\title{
Dysphagia in unilateral cerebral lesions
}

\author{
J. C. MEADOWS
}

\author{
From the National Hospital, Queen Square, London
}

SUMMARY Dysphagia may in rare instances be a presenting feature of unilateral cerebral lesions. Normally, bilateral lesions are necessary to cause neurological disturbance of swallowing but there appear to be occasional subjects where this is not so. Cases are presented and the literature reviewed to illustrate this. The dysphagia may occur in isolation and is not necessarily accompanied by dysarthria, facial apraxia, or obvious paralysis. The lesion may be on either side and usually involves the low posterior frontal region. Cases that have come to necropsy have shown involvement of the lowest part of the precentral gyrus or the posterior part of the inferior frontal gyrus.

When dysphagia occurs in neurological disease it is usually accompanied by dysarthria, and, whatever level of the central nervous system is involved, it is generally held that a bilateral disturbance must be present. So far as descending pathways are concerned, this means that a single structural lesion is likely to cause dysphagia only if it affects the brain-stem, where pathways on the two sides are in close proximity. In general, it can be assumed that if there is a supratentorial cause for dysphagia, this must be bilateral and is therefore probably either vascular or degenerative.

The purpose of this paper is to point out that in rare instances a unilateral cerebral lesion may be responsible for dysphagia. Three cases are described in which dysphagia was an initial symptom of a space-occupying lesion affecting one cerebral hemisphere, and a number of other relevant cases are discussed. It is not intended to imply that this is a common occurrence but merely to indicate that exceptions do occur to what is generally a sound rule.

\section{CASE 1}

(A.56173) A 70 year old right-handed man began to notice difficulty in swallowing liquids some 10 months before admission. This became worse so that a cup of tea was cold before he had finished it and a half-pint $(265 \mathrm{ml}$.) glass of beer took up to half an hour to drink. He said it was 'as if a flap came down at the back of the throat'. Solids presented rather less difficulty. He was seen first at the Royal National
Throat, Nose and Ear Hospital where a barium swallow showed no structural abnormality but 'considerable hesitancy in swallowing' was described. Direct laryngoscopy was normal.

He was admitted to the National Hospital. There he complained of occasional urgency and incontinence of faeces with less marked urgency of micturition for about two months. He felt depressed. Although both he and his family at first denied any intellectual change, his wife subsequently stated that he had recently started to sleep in the afternoons, was coming home late, and did not always attend for meals.

General examination was normal. Neurological examination four months before admission had been reported normal but in the intervening period he had developed a mild left supranuclear facial palsy. The only other abnormal finding was that the right plantar response was less clearly flexor than the left.

Clinical testing of intellect was normal. His speech was fluent and there was no difficulty in naming or in repetition. Articulation was at all times normal and there was no facial apraxia; he could whistle, blow out his cheeks to command, show his teeth, put out his tongue, and imitate sounds articulated by the examiner. The palate moved briskly and centrally on both phonation and reflex testing, and tongue movements were full and rapid to command.

When he attempted to drink from a glass of water, this was done slowly, in single gulps which were retained in the mouth often for many seconds before being swallowed. Cinefluorography showed that there was great hesitancy in initiating the pharyngeal phase of swallowing, contrast (whether liquid or semi-solid) lapping incoordinately back and forth 


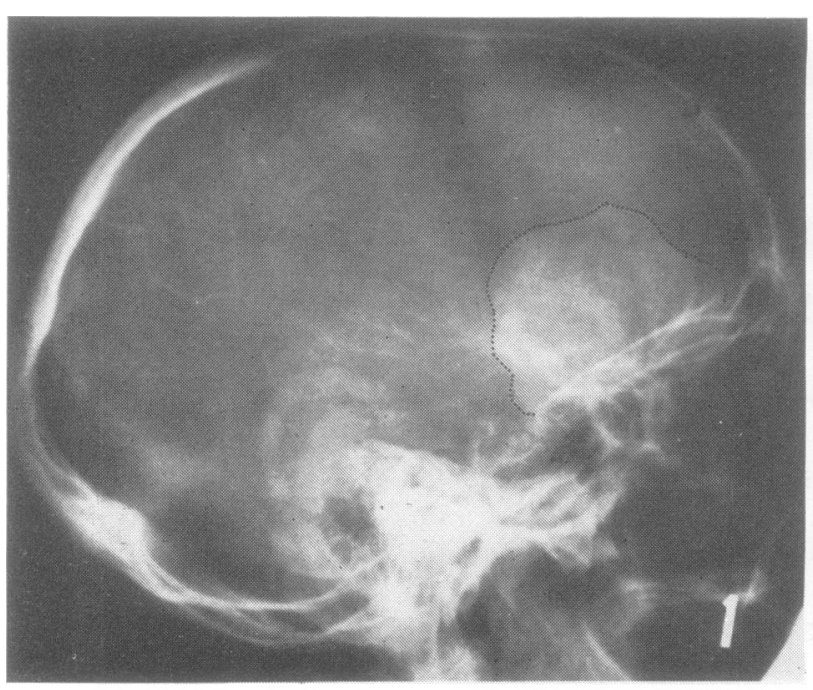

(a)

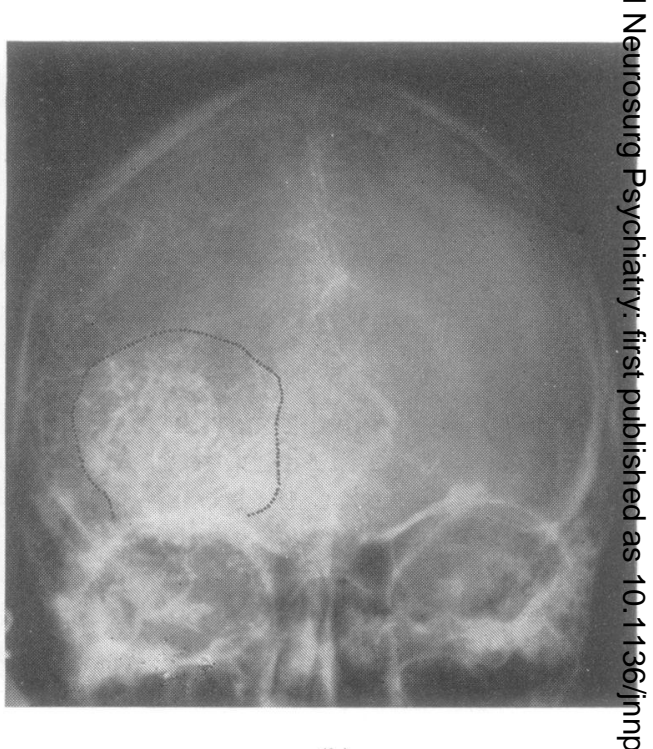

(b)

FIG. 1. Tumour blush outlined on right carotid angiogram in case 1 .

within the oral cavity while the palate also moved irregularly until, after a variable period, it suddenly elevated to close off the nasopharynx and the tongue simultaneously propelled the contrast into the pharynx. While lying on his back, so that gravity carried the contrast into the pharynx, there was no delay. These studies were kindly carried out at University College Hospital by Dr. D. G. Shaw.

Psychological testing (Wechsler) revealed a Verbal IQ of 127 and a Performance IQ of 116. Radiographs of the skull and chest were normal. An electroencephalogram (EEG) showed some mainly postcentral excess of slow activity, with right-sided emphasis. A technetium scan showed increased uptake in the right subfrontal area. Right carotid angiography demonstrated a large meningioma arising from the right sphenoidal ridge (Fig. 1a, b) and causing a shift of the pericallosal artery and internal cerebral vein.

At operation the meningioma was mushrooming deeply into the brain but was removed successfully after resection of some frontal cortex anterolaterally. Histology confirmed a typical meningioma. Within a few days he was ambulant but the dysphagia was unchanged. He died suddenly from a pulmonary embolus six days postoperatively.

Post mortem examination revealed a depression on the posterolateral aspect of the inferior part of the right frontal lobe of the brain, partially filled with blood clot. Much of the posterior part of the inferior frontal gyrus was destroyed but at posterior end there was a narrow strip which âp peared healthy, separating the necrotic area from ghष lowest part of the precentral gyrus. The precentrat gyrus itself appeared to be intact but was examined microscopically. There was some tentoriad herniation on the right and some subfalcine hernia tion. The brain was sectioned throughout and the extent of the local cerebral damage can be seen in the frontal slices (Fig. 2). No other focal lesions were seen. Microscopic examination of the nucleus ambiguus on each side revealed no abnormality.

\section{CASE 2}

(M.H. A.6686) This patient was under the care on Professor R. W. Gilliatt at the Middlesex Hospital

A 36 year old right-handed storeman was admittew for investigation in May 1972. His right eye had beeis enucleated after an illness in early childhood. Other wise he had been well until two years previously whero he began complaining of intermittent headache, a first occipitally and later either bitemporally or con? fined to the right temple. For about a year he had had difficulty in swallowing liquids. He would take smale sips and would then have great difficulty in trans ferring the liquid to the back of his throat; it would regurgitate into his nose or even be ejected out of his mouth. This dysphagia varied in severity. On occas N ions it would take 15 to 20 minutes to drink a glas 

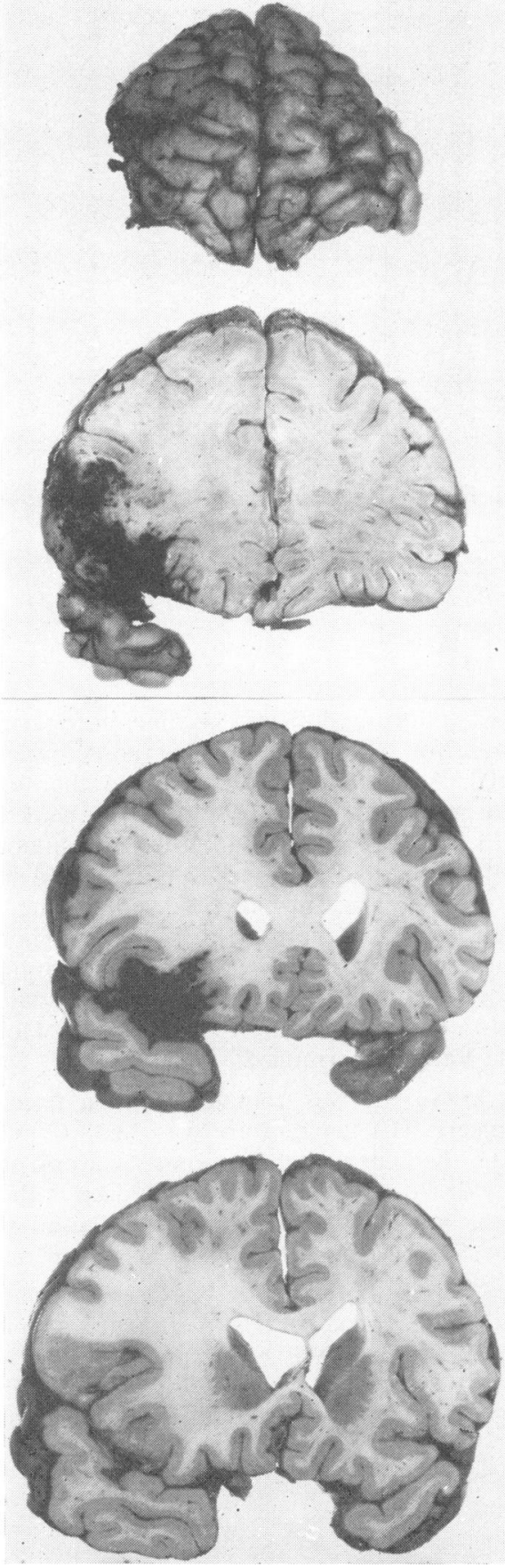

FIG. 2. Successive sections through the frontal lobes in case 1, the most posterior being at the level of the heads of the caudate nuclei. of water, and at one time it reached such severity that he telephoned his doctor in a panic. In contrast, swallowing of solids was little if at all affected. For three months before admission he had noticed impaired concentration and felt that his balance was imperfect. There had been a single episode of paraesthesiae in the left hand lasting a few minutes.

He had been examined by neurologists on several occasions but no abnormality was found until April 1972 when it was noted that saliva was pooling at the back of his mouth and that the palatal and pharyngeal reflexes were absent, although sensation was preserved. On phonation the palate moved well but slightly to the left of the midline. When admitted a month later there was thought, in addition, to be evidence of mild intellectual impairment and also minimal slurring of speech. Early papilloedema was noted. A mild upper motor neurone facial weakness was present on the left, and the right plantar response was thought by some observers to be extensor. Ten-
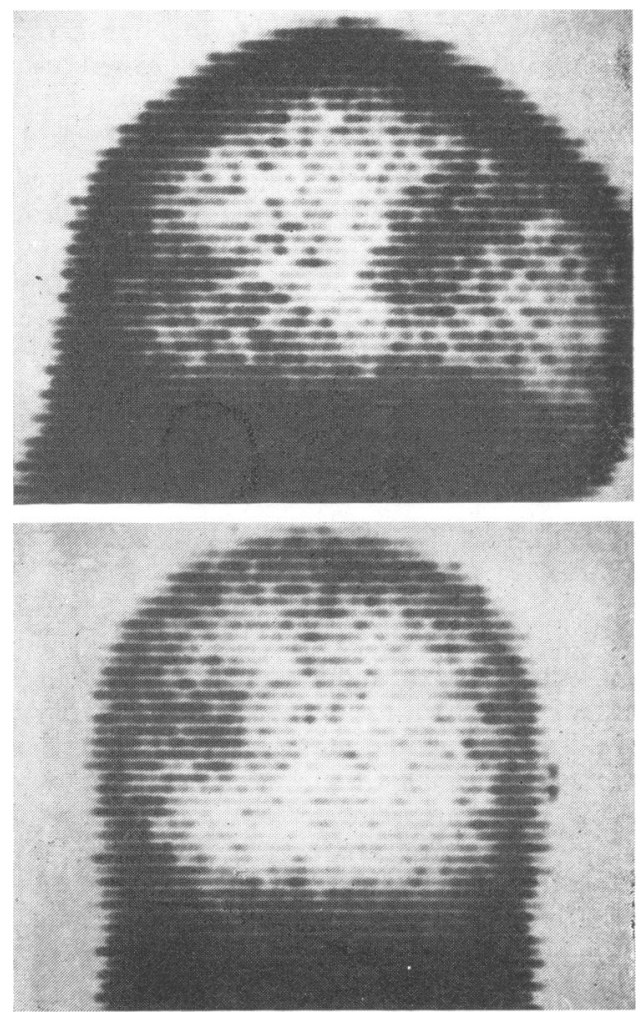

FIG. 3. Radioisotope brain scan demonstrating tumour site in case 2 . 


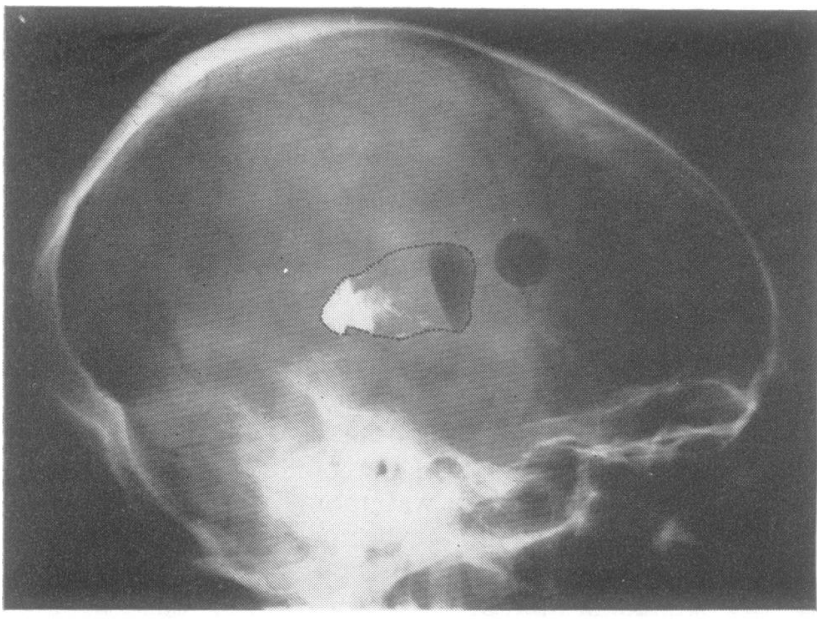

(a)

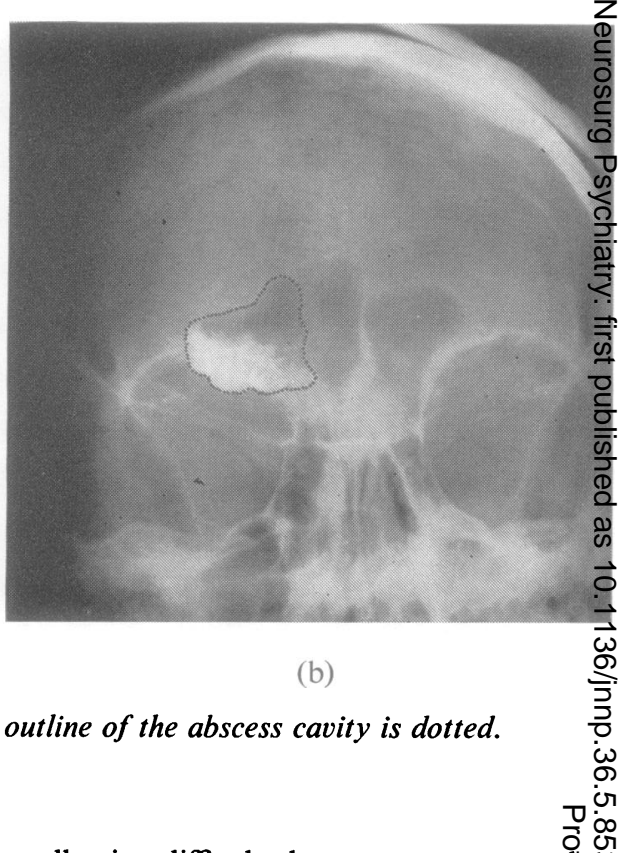

The swallowing difficulty became more severe no cause for it was found. A low-grade pyrexia fade veloped. After 10 days it was noted that the Peft plantar response was extensor and over the next fout days he became confused with a left hemianopia \&nd flaccid hemiplegia. His neck was rigid and lun puncture revealed turbid fluid containing 2,875

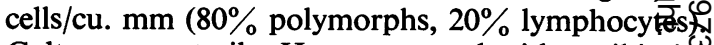
Culture was sterile. He was treated with antibiotics but continued to deteriorate and three days later was comatose. Urgent transfer to the National Hospitas Maida Vale, was arranged.

On arrival he was comatose with a flaccid le hemiplegia. His temperature was $38.3^{\circ} \mathrm{C}\left(101^{\circ} \mathrm{F}\right.$ and he had bronchopneumonia. Carotid angiळ graphy disclosed an avascular mass deep in the right cerebral hemisphere which proved to be an absces due to Staphylococcus aureus. A pyogram outlined the abscess cavity (Fig. 4).

With medical and surgical treatment his condition improved progressively. Two months later, when finally discharged, he was swallowing normally ang eating well but had a residual right hemiparesis.

Since being alerted to the fact that dysphagia mationAL CASES occur in exceptional patients with unilateral cerebrap lesions, the author has seen, in addition to thosed reported above, three further cases where difficulty in swallowing has been a minor feature, and they arE siderable periods' before swallowing began.

Radiographs of the chest were normal. A barium swallow showed no structural abnormality but it was noted that 'the patient's difficulty appeared to be mainly in the mouth, barium remaining for con-

A 59 year old right-handed man was admitted to in swallowing, saying that food stayed in his mouth. Three years earlier he had had a Polya gastrectomy examination was reported as normal and no defect was noted in his speech. 
reported at this point simply to draw attention to the site of the lesions. All were seen at the National Hospital, Maida Vale. In two patients with large frontal mass lesions, dysphagia occurred (eclipsed by forgetfulness and frontal gait disturbance) for some months before diagnosis. One had a large right pterion meningioma (M.V.H. 68957), the other a right frontal arachnoid cyst (M.V.H. 70400); neither showed overt pseudobulbar palsy but the former patient had mild dysarthria. Another patient (M.V.H. 72254) developed first right facial weakness and then dysphasia and focal sensory seizures affecting the right arm. He finally developed severe dysphagia requiring nasogastric feeding. A malignant glioma of the left posterior frontal region was found on investigation.

\section{DISCUSSION}

There are a few previously reported cases of severe dysphagia occurring in patients with lesions confined to one cerebral hemisphere. Bastian (1898) described a patient in whom there was sudden onset of aphasia and right hemiplegia associated with difficulty in swallowing and in protruding the tongue, both of which subsequently improved. The patient finally died and at necropsy the brain was found to be healthy 'except at two limited spots, of which the chief was the posterior part of the third frontal convolution on the left side. Here was a softened and almost diffluent patch, about three-quarters of an inch in breadth, reaching from the highest part of the third convolution backwards and downwards to the fissure of Sylvius. The softened part was not actually the most posterior part of the convolution for there was a narrow, unsoftened strip between it and the ascending frontal convolution. On cutting into the brain a second small patch of softening was seen in the centre of the left hemisphere, external to and rather above the corpus striatum, and extending towards the posterior extremity of the fissure of Sylvius.'

Pussep and Levin (1923) reported a woman who developed severe dysphagia and anarthria abruptly, and had to initiate swallowing by pushing food manually to the back of the throat, throwing her head back until food came into contact with the pharynx, whereupon swallowing proceeded normally. Necropsy showed two areas of softening, one in the left supramarginal gyrus, the other in the lower part of the left precentral gyrus. A similar patient, described by Tuch and Nielsen (1941) developed sudden complete aphagia and anarthria, being unable to swallow even saliva. There was apraxia of the facial musculature but the only other neurological sign described was a slight droop of the right side of the mouth. Necropsy revealed softening of the lower part of the left pre- and post-central gyri.

Unlike these patients, two of the present three cases had dysphagia as the sole initial symptom. The other case had a past history of headache but again dysphagia was severe and for some time led to a mistaken diagnosis of posterior fossa tumour. Cases 1 and 2 were very similar clinically. In both there was selective dysphagia for fluids, facial apraxia was absent, the tongue moved fully and freely to command, the jaw jerk was not increased, and the palate elevated well on phonation. However, case 2 differed in that the palatal and pharyngeal reflexes were absent bilaterally, although sensation was preserved. This was not recorded in the other cases mentioned above and the reason for it is not clear. The lesion in both cases 1 and 2 was a tumour in the right postero-inferior frontal region. Case 1 died of pulmonary embolus six days postoperatively and the brain was examined at necropsy. The findings were complicated by the effects of operation, but the main site of damage was the posterior part of the inferior frontal gyrus and the white matter deep to it. The precentral gyrus did not look damaged macroscopically, but the meningioma as demonstrated radiologically (Fig. 1) almost certainly encroached close onto the lowest part of this gyrus.

In case 3 , the lesion (an abscess) was more deeply placed. However, this patient was not examined by the author or investigated neurologically until some weeks after the initial onset of dysphagia, by which time he had lapsed into coma. There had probably been considerable extension of the abscess during this period. It was the only case where the lesion was deeply placed and, since the first symptom was a similar difficulty in the initial stage of swallowing to cases 1 and 2, it is possible that the abscess may have interrupted either afferent or efferent con- 
nections with the cortical area affected in the other patients.

The low posterior frontal region was involved on one or other side in all the other cases, but the precise site of the lesion requires further discussion. The necropsy findings in case 1 showed that the precentral gyrus was macroscopically spared and that the major site of damage was further forward in the inferior frontal gyrus. However, it is likely that in life the lowest part of the precentral gyrus was subject to considerable pressure and distortion. Necropsies were not performed in the other tumour cases and the available information is not sufficient for precise localization within this general area. On the other hand the three cases with vascular disease had discrete cortical lesions at necropsy. In two cases the lowest part of the precentral gyrus was involved but in the third the lesion was apparently confined to the inferior frontal gyrus. If these descriptions are to be believed it appears that a lesion either of the precentral gyrus or the posterior part of the inferior frontal gyrus may cause dysphagia. However, only in Bastian's case was the inferior frontal gyrus said to be involved in isolation.

Penfield and Jasper (1954) have shown that stimulation of the lower part of either precentral gyrus at operation causes swallowing, vocalization, and movement of the face and jaw, and this accords with the above localization. However, there is normally bilateral representation of the cranial musculature in the primary motor cortex, and bilateral lesions are usually required to cause permanent disturbance of speech or swallowing. Bilateral, symmetrical cortical lesions are rare in man, but aphagia was reported, for example, by Foix, Chavany, and Marie (1926) in a case where softening of the lowest part of both precentral gyri and the posterior part of the inferior frontal gyri was found at necropsy. Similarly, bilateral corticobulbar tract lesions in cerebrovascular disease cause pseudobulbar palsy, where dysphagia is a prominent symptom.

In contrast, unilateral lesions are not usually associated with significant dysphagia. Strokes causing hemiplegia occasionally give rise to transient hesitancy in swallowing but this only very rarely causes the patient concern. However, Penfield and Jasper (1954) found that significant dysphagia may occur transiently after unilateral cortical ablations: the patient may 'seem to forget how to swallow for a few days ... anc mouth and tongue may be used awkwardly for at time in forming words, after a lesion of the lowef Rolandic region, even on the side opposite te the localization of speech'.

On the basis of these observations it therefore seems likely that the dysphagia seen in the present patients relates to damage at or close to $\overrightarrow{\vec{s}}$ the lowest part of the precentral gyrus. Af alternative explanation in the tumour cases might be that dysphagia is a false localizing feature due in some way to raised intracrania $\mathbb{B}$ pressure or distortion of intracranial structures but if this were so it is remarkable that the site of the lesions was always approximately the sameIt is possible that a tumour at this site causes unie灬 lateral damage and that contralateral damage occurs as a false localizing feature, perhaps b pressure against the opposite tentorial edge. It is notable that the plantar response ipsilaterally tor the tumour in cases 1 and 2 was questionabis abnormal, which would support this view. Buff it cannot explain the vascular cases. In the vasou? lar cases, unsuspected damage on the opposit side might be postulated but this was not founi in the cases reported above that came to necropis We are left with the suggestion that there are rare individuals in whom the pathways in a singey hemisphere are incapable of alone supporting the swallowing process.

Tuch and Nielsen (1941) discussed thei patient under the title 'Apraxia of swallowing However, the use of the term 'apraxia' in this context is arguable and it may be more correct to regard the dysphagia as a more elementarg motor disturbance. Dysphagia is not usually feature of the more generalized apraxia of the bulbar musculature known as facial apraxia Thus, facial apraxia typically affects the tongue which cannot be protruded to command yet ma\% be protruded spontaneously a few moments. later to lick the lips. No such apraxia occurreg in cases 1 and 2, for example, yet in case incoordinated movement of the tongue wa demonstrated radiologically during attemptes swallowing. It is true that dysphagia and facial apraxia may coexist as in Tuch and Nielsenfo case, but it is questionable whether they ate manifestations of the same disorder rather thas merely neighbourhood symptoms. The fact that 
dysphagia may occur with either right or leftsided lesions, which is very rarely the case with facial apraxia, casts further doubt on their relationship.

This leads us to a consideration of the anatomical pathways subserving these functions. The pathway for linguofacial movements to command is linked to cerebral speech dominance and its likely route from Wernicke's area forward in the arcuate fasciculus to the association cortex anterior to the left face area and thence to both primary motor representations has been discussed by Geschwind (1965). Interruption anywhere from Wernicke's area to the left premotor cortex will cause facial apraxia. On the other hand, the central pathway for swallowing is normally bilateral, is not linked to speech, and at its simplest may consist of little more than an afferent projection from the periphery to the primary sensory cortex bilaterally, thence by the abundant connections known to exist in primates (Bailey, Bonin, and McCulloch, 1940; Jones and Powell, 1969) to the primary motor cortex (perhaps via premotor cortex) and finally to the pyramidal tracts. Premotor cortex may be involved, for bilateral excision of the inferior frontal gyri caused persistent dysphagia and dysarthria in a patient reported by the ColumbiaGreystone associates (1949). Also, the limited description of the necropsy findings in Bastian's case suggests that the primary motor region was spared. However, it cannot be certain in these cases that dysphagia was not due to secondary damage to the motor pathway, for bilateral excisions of the anterior, middle, and posterior parts of the inferior frontal gyri in other patients in the Columbia-Greystone study did not cause dysphagia.

The anencephalic infant may suckle successfully as long as the brain-stem is intact (Gamper, 1926; Utter, 1928), indicating that cortical function is not essential for swallowing. However, the situation in the infant is different from the adult, for the teat or nipple extends well back into the oral cavity so that milk is carried directly by the sucking reflex into the pharynx, from where reflex swallowing follows. In the adult, food has to be propelled actively through the oral cavity, the tongue pressing upward and forwards against the hard palate so that the bolus is squeezed backward like toothpaste from a tube
(Ardran and Kemp, 1951). At the same time, the nasopharynx is closed off. It is this process, presumably learned during weaning, that is defective in the present cases. It is a stereotyped semi-automatic act which once learned may require little cortical intervention other than in its initiation, some control being necessary to reject unpalatable items or to deal with the varied foods encountered in everyday life; liquids are swallowed without delay but solids require first a variable degree of mastication. Why swallowing of liquids should be so much more affected in at least some of the cases of dysphagia associated with unilateral cerebral lesions is not clear but possibly these act as a less effective stimulus in initiating the process.

Finally, it is interesting to note that, according to Heilbronner (quoted by Nathan, 1947), reflex swallowing may persist when higher control is defective. He claims that the advanced 'apractic' who cannot swallow normally retains the sucking reflex and can be satisfactorily nourished by bottle like an infant.

I am grateful to Dr. J. N. Blau, Professor R. W. Gilliatt, and Professor John Marshall for permission to report these cases, to Dr. P. W. Nathan for his helpful comments on the manuscript, and to Professor William Blackwood for his help with the neuropathological aspects.

\section{REFERENCES}

Ardran, G. M., and Kemp, F. H. (1951). The mechanism of swallowing. Proceedings of the Royal Society of Medicine, 44, 1038-1040.

Bailey, P., Bonin, G. von, and McCulloch, W. S. (1950). The Isocortex of the Chimpanzee. University of Illinois Press: Urbana.

Bastian, H. C. (1898). A Treatise on Aphasia and Other Speech Defects, p. 87. Lewis: London.

Columbia-Greystone Associates (1949). Selective Partial Ablation of the Frontal Cortex. Edited by F. A. Mettler. Hoeber: New York.

Foix, Ch., Chavany, J.-A., and Marie, J. (1926). Diplégie facio-linguo-masticatrice d'origine cortico sous-corticale sans paralysie des membres. Revue Neurologique, 1er sem., 214-219.

Gamper, E. (1926). Bau und Leistungen eines menschlichen Mittelhirnwesens (Arhinencephalie mit Encephalocele). Zugleich ein Beitrag zur Teratologie und Fasersystematik. Zeitschrift gesamte fur die Neurologie und Psychiatrie, 102, 154-235.

Geschwind, N. (1965). Disconnexion syndromes in animals and man. Brain, 88, 585-644.

Jones, E. G., and Powell, T. P. S. (1969). Connexions of the 
somatic sensory cortex of the rhesus monkey. 1. Ipsilateral cortical connexions. Brain, 92, 477-502.

Nathan, P. W. (1947). Facial apraxia and apraxic dysarthria. Brain, 70, 449-478.

Pussep, L., and Levin, L. (1923). Zur Frage der Störungen des Schluckens von apraktischem Charakter (Aphago- praxie). Zeitschrift für die gesamte Neurologie und Psychiatrie, 87, 441-450.

Tuch, B. E., and Nielsen, J. M. (1941). Apraxia of swallowing. Bulletin of the Los Angeles Neurological Society, 6, 52-54.

Utter, O. (1928). Ein Fall von Anenzephalie. Acta Psychiatrica et Neurologica, 3, 281-318. 